\title{
Aeromonas spp asociada a enfermedad diarreica aguda en Cuba: estudios de casos y controles
}

\author{
Laura Bravo, Anabel Fernández, Fidel Á. Núñez, Luis A. Rivero, Margarita Ramírez, \\ Adalberto Águila, Yudith Ledo, Yanaika Cruz y Jenny Hernández
}

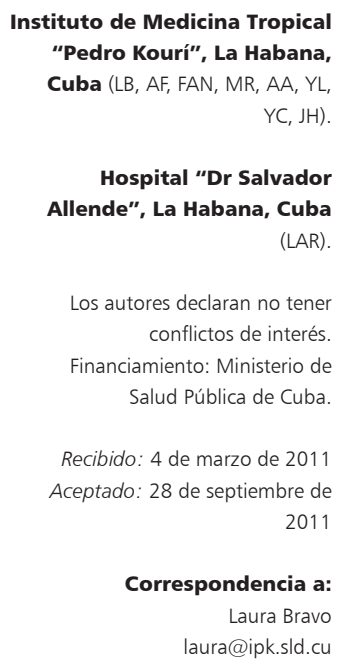

Instituto de Medicina Tropical "Pedro Kourí", La Habana, $Y C, J H)$ compared between both groups, we found that probability to isolate this specie was significantly higher in cases than in controls $(\mathrm{OR}=4.48,95 \%$ IC: $3.05-6.60 ; P<0.001)$. The Aeromonas species more frequently isolated
were $A$. caviae, $A$. hydrophila, and $A$. veronii bv sobria. Other enteric pathogens detected in children with diarrhea
were: Shigella spp in $418(18 \%)(P<0.0001)$, Salmonella spp in $53(2.3 \%)(P<0.01)$, and enteropathogenic $E$. were $A$. caviae, $A$. hydrophila, and $A$. veronii bv sobria. Other enteric pathogens detected in children with diarrhea
were: Shigella spp in $418(18 \%)(P<0.0001)$, Salmonella spp in $53(2.3 \%)(P<0.01)$, and enteropathogenic $E$.
coli in $58(2.49 \%)(P<0.05)$.

Key words: Intestinal infection, Aeromonas, case-control study. were $A$. caviae, $A$. hydrophila, and $A$. veronii bv sobria. Other enteric pathogens detected in children with diarrhea
were: Shigella spp in $418(18 \%)(P<0.0001)$, Salmonella spp in $53(2.3 \%)(P<0.01)$, and enteropathogenic $E$.
coli in $58(2.49 \%)(P<0.05)$.

\section{ntroducción}

Las enfermedades infecciosas son uno de los principales problemas a los que se enfrentan los países en vías de desarrollo. En Cuba, la población rebasa los 11 millones de 
si a través de las características particulares del cuadro clínico que producen es posible inferir si son causadas por agentes bacterianos. Por otro lado, los estudios etiológicos permiten implementar medidas de control y prevención 9 .

Las especies patógenas de Aeromonas con mayor implicación clínica en casos de diarreas son: Aeromonas hydrophila, Aeromonas veronii biovar sobria, Aeromonas caviae y, en menor proporción, Aeromonas veronii biovar veronii, Aeromonas jandae y Aeromonas schubertii. Algunas de estas especies han estado asociadas con la presencia de enterotoxinas, citotoxinas y con marcadores fenotípicos específicos relacionados con la enfermedad gastrointestinal en el hombre ${ }^{3,6}$.

Por lo antes expuesto y por la importancia que reviste a nivel nacional e internacional la vigilancia microbiológica y epidemiológica de los microorganismos pertenecientes a este género, analizamos los resultados de estudios sobre Aeromonas realizados en Cuba entre los años 1985 y 2005, utilizando en todos los estudios la misma metodología diagnóstica, con el objetivo de comparar la frecuencia de positividad de los microorganismos pertenecientes al género Aeromonas entre casos y controles e identificar las especies de este género circulantes en nuestro país en ese período de tiempo.

\section{Materiales y Métodos}

Entre los años 1985 y 2005 se llevaron a cabo varios estudios de casos y controles en los que se realizó la búsqueda activa en las heces de microorganismos del género Aeromonas, utilizando la misma metodología diagnóstica, y cuya muestra total estuvo constituida por 2.322 niños bajo
5 años de edad, que ingresaron por enfermedad diarreica aguda, con menos de 72 horas de evolución, procedentes de diferentes hospitales pediátricos del país, y que no habían recibido tratamiento con antimicrobianos. Se seleccionó como grupo control un total de 2.072 niños con edades y áreas geográficas similares, que acudieron a los hospitales correspondientes en ese mismo período de tiempo, pero por otras enfermedades, que no hubieran experimentado episodio diarreico alguno en los meses precedentes, ni hubieran recibido tratamiento antimicrobiano por lo menos 10 días antes de obtener la muestra. En la Figura 1 se muestran las provincias del país, y los años en que se realizaron los estudios de casos y controles en diferentes hospitales pediátricos.

A cada niño se le realizó hisopado rectal transportándose la muestra en medio de Cary-Blair, conservada a temperatura ambiente y procesada en los centros centinelas en un tiempo que no excedió a las 48 horas ${ }^{10}$.

Las muestras conservadas en el medio de Cary-Blair fueron sembradas en los medios de agar MacConkey (MK) y agar Salmonella-Shigella (SS) para investigar la presencia de Aeromonas, Salmonella, Shigella y E. coli enteropatógena e incubados en aerobiosis a $37^{\circ} \mathrm{C}(18-24$ h). Simultáneamente, las muestras fueron transferidas a los medios de enriquecimento de agua peptona alcalina $(\mathrm{pH} 8,6)$ que fue incubada a $37^{\circ} \mathrm{C}$ en condiciones de aerobiosis por 18 horas, así como a caldo selenito, el cual fue incubado durante 14 horas en las mismas condiciones de temperatura y atmósfera. Transcurrido el tiempo de incubación, se seleccionaron cuatro colonias por placa (translúcidas o no, con o sin presencia de sulfuro ferroso) las que se inocularon por punción y estrías en los medios de diferenciación primaria: agar hierro y dos azúcares de

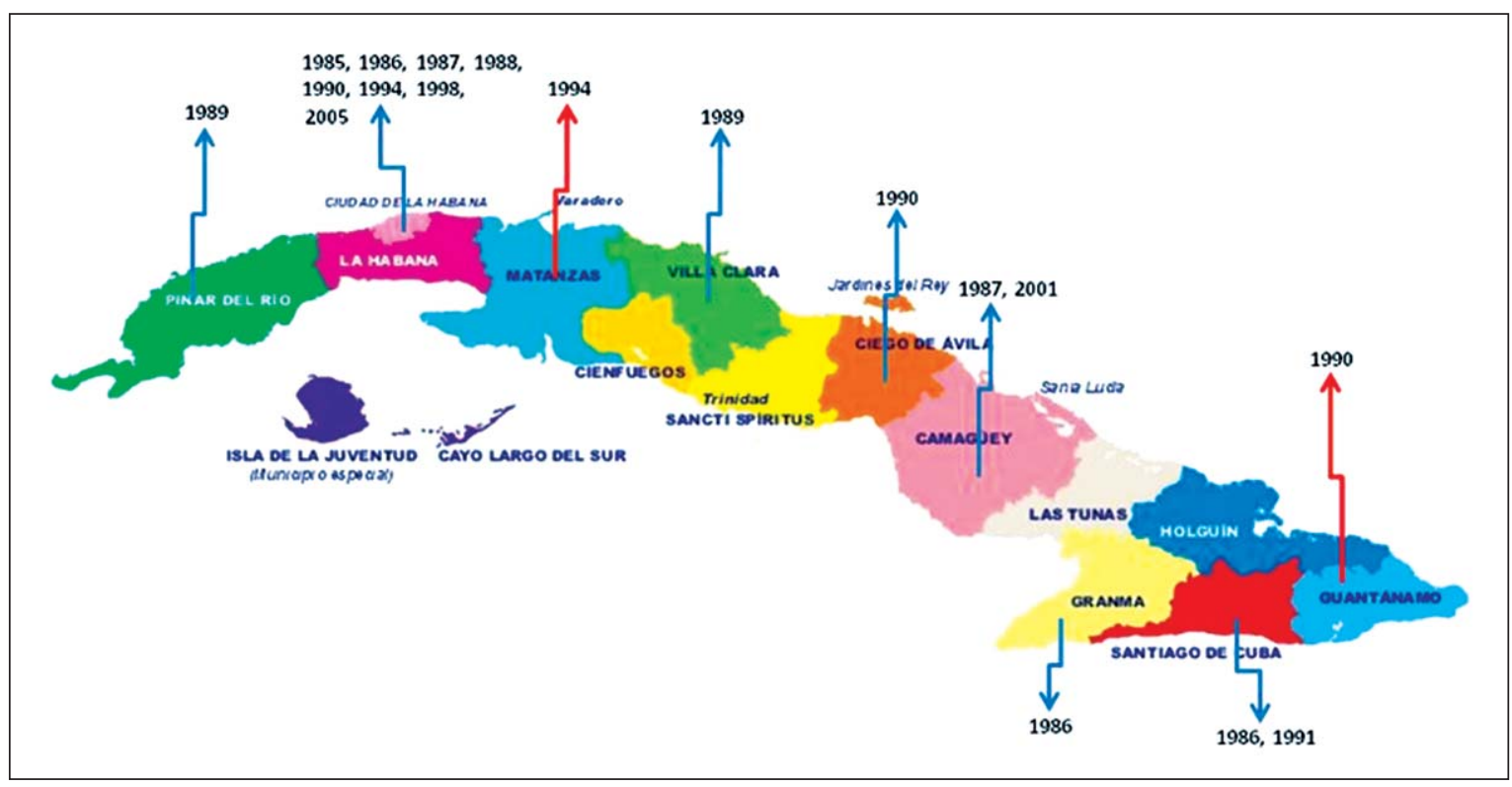

Figura 1. Mapa de las provincias y los años en que se realizaron estudios de casos y controles sobre Aeromonas spp en Cuba. 
Tabla 1. Estudios de casos y controles sobre Aeromonas spp realizados entre los años 1985 y 2005 en Cuba

\begin{tabular}{|c|c|c|c|c|c|c|}
\hline \multirow[t]{3}{*}{ Año } & \multirow[t]{3}{*}{ Lugar } & \multirow{2}{*}{\multicolumn{2}{|c|}{$\begin{array}{c}\text { Casos con } \\
\text { diarrea }\end{array}$}} & \multicolumn{3}{|c|}{$\begin{array}{c}\text { Controles sin } \\
\text { diarrea }\end{array}$} \\
\hline & & & & \multicolumn{3}{|c|}{ Total infectados* } \\
\hline & & & n $\quad(\%)$ & & $\mathrm{n}$ & \\
\hline 1985 & Hospital Pediátrico Marfán, La Habana & 100 & $0 \quad(0,0)$ & 100 & 0 & $(0,0)$ \\
\hline 1986 & Hospital Pediátrico de Centro Habana, La Habana & 200 & $8(4,0)$ & 200 & 0 & $(0,0)$ \\
\hline 1986 & Hospital Pediátrico "General Milanês", Bayamo & 200 & $18(9.0)$ & 200 & 17 & $(8,5)$ \\
\hline 1986 & Hospital Pediátrico Sur, Santiago de Cuba & 100 & $7(7,0)$ & 100 & 2 & $(2,0)$ \\
\hline 1987 & Hospital Pediátrico del Cerro, La Habana & 100 & $13(13,0)$ & 50 & 0 & $(0,0)$ \\
\hline 1987 & Hospital Pediátrico de Camagüey & 100 & $4(4,0)$ & 100 & 0 & $(0,0)$ \\
\hline 1988 & Hospital Pediátrico de Centro Habana, La Habana & 140 & $19(13,5)$ & 140 & 3 & $(2,1)$ \\
\hline 1988 & Hospital Pediátrico "Pedro Borras", La Habana & 150 & $21(14,0)$ & 50 & 0 & $(0,0)$ \\
\hline 1988 & Hospital Pediátrico de Camagüey & 100 & $3(3,0)$ & 100 & 0 & $(0,0)$ \\
\hline 1989 & Hospital Pediátrico "Pepe Portilla", Pinar del Río & 150 & $19(12,6)$ & 150 & 4 & $(2,7)$ \\
\hline 1989 & Hospital Pediátrico de Villa Clara & 100 & $6(6,0)$ & 100 & 2 & $(2,0)$ \\
\hline 1990 & Hospital Pediátrico de Ciego de Ávila & 50 & $6(12,0)$ & 50 & 2 & $(4,0)$ \\
\hline 1990 & Hospital Pediátrico de Guantánamo & 100 & $10(10,0)$ & 100 & 4 & $(4,0)$ \\
\hline 1990 & Hospital Pediátrico de Centro Habana, La Habana & 100 & $2(2,0)$ & 50 & 0 & $(0,0)$ \\
\hline 1991 & Hospital Pediátrico Sur, Santiago de Cuba & 100 & $8(8,0)$ & 50 & 1 & $(2,0)$ \\
\hline 1994 & Hospital Pediátrico "William Soler", La Habana & 132 & $8(6,06)$ & 132 & 0 & $(0,0)$ \\
\hline 1994 & Hospital Pediátrico de Matanzas & 100 & $4(4,0)$ & 100 & 0 & $(0,0)$ \\
\hline 1998 & Hospital Pediátrico "William Soler", La Habana & 100 & $4(4,0)$ & 100 & 0 & $(0,0)$ \\
\hline 2001 & Hospital Pediátrico de Camagüey & 100 & $3(3,0)$ & 100 & 0 & $(0,0)$ \\
\hline \multirow[t]{2}{*}{2005} & Hospital Pediátrico del Cerro, La Habana & 100 & $3(3,0)$ & 100 & 0 & $(0,0)$ \\
\hline & Total de hospitales pediátricos & 2.322 & $166(7,15)$ & 2.072 & 35 & $(1,76)$ \\
\hline
\end{tabular}

\begin{tabular}{|c|c|c|}
\hline Especies de Aeromonas & $\mathbf{n}$ & $\%$ \\
\hline A. caviae & 57 & 34,3 \\
\hline A. hydrophila & 51 & 30,7 \\
\hline A. veronii bv sobria & 36 & 21,6 \\
\hline A. trota & 7 & 4,2 \\
\hline A. veronii bv veronii & 5 & 3,2 \\
\hline A. schubertii & 2 & 1,2 \\
\hline A. jandaei & 1 & 0,6 \\
\hline Aeromonas spp & 7 & 4,2 \\
\hline Total & 166 & 100 \\
\hline
\end{tabular}

Kligler (AHK), y agar hierro y lisina (AHL), incubados todos en aerobiosis a $37^{\circ} \mathrm{C}$, durante 18 a $24 \mathrm{~h}^{11,12}$.

A todas las cepas con aspecto característico de pertenecer al género Aeromonas se les investigó la presencia de la enzima citocromo oxidasa, según el método de Kovacs. Posteriormente, a las colonias portadoras de genes para la enzima citocromo-oxidasa, se les realizaron los estudios fisiológicos para ubicarlas en género y especie, aplicando los sistemas de identificación fenotípica: Aerokey II (método que emplea siete pruebas bioquímicas diferentes) y Aeroesquema (método que consiste en seis pruebas bioquímicas e identifica no sólo especies de Aeromonas, sino que también Vibrio y Plesiomonas shigelloides) ${ }^{13,14}$. A todas las colonias negativas para la enzima citocromo oxidasa, se les realizó estudios complementarios para determinar la especie, según los métodos descritos en el Manual de Bacteriología Sistemática de Bergey de $2005^{12}$.

Para los análisis estadísticos se calculó la razón de disparidad puntual $\mathrm{u}$ oportunidad relativa (OR) con sus respectivos intervalos de confianza al $95 \%$ con el objetivo de comparar la frecuencia de positividad en los casos de diarrea y en los controles. También se empleó la prueba de proporciones para comparar los porcentajes de positividad de las diferentes especies de Aeromonas. En todos los casos las diferencias se consideraron estadísticamente significativas cuando se obtuvo un valor de $P<0,05$. Todos los análisis se desarrollaron empleando el paquete de programas EPIINFO, versión 6.04 y Epidat versión 3.1 $1^{15}$.

\section{Resultados}

De los 2.322 niños que presentaron diarrea, Aeromonas spp fue aislada en $166(7,15 \%)$, y en $35(1,76 \%)$ de los controles. Al analizar la positividad para Aeromonas entre casos y controles para la totalidad de los estudios realizados en Cuba entre 1985 y 2005, la probabilidad de diagnosticar este microorganismo fue 4,48 veces mayor en pacientes pediátricos con diarrea (casos) que los controles (OR = 4,48; IC al 95\%: 3,05-6,60; $P<0,001$ ) (Tabla 1).

$\mathrm{Al}$ aplicar los esquemas Aerokey II y Aeroesquema se identificó a 95,2\% de las especies pertenecientes al género Aeromonas. La distribución entre ellas resultó la siguiente: A. caviae 57 (34,3\%); A. hydrophila $51(30,7 \%)$; A. veronii bv sobria $36(21,6 \%)$; A. trota $7(4,2 \%) ; A$. veronii bv veronii 5 (3,2\%); A. schubertii $2(1,2 \%)$; $A$. jandaei $1(0,6 \%)$ y Aeromonas spp $7(4,2 \%)$ (Tabla 2$)$. Se encontró diferencias entre las frecuencias de positividad por especies entre los 166 aislados estudiados $\left(\chi^{2}=210,92\right.$; $P<0,001$ ), aunque las especies más frecuentes en este período analizado fueron A. caviae y $A$. hydrophila.

Otros enteropatógenos aislados en niños que presentaban diarrea fueron: Shigella spp. en $418(18 \%)$ $(P<0,0001)$, Salmonella spp en $53(2,3 \%)(P<0,01)$ y Escherichia coli enteropatógena en $58(2,49 \%)(P<0,05)$. 


\section{Discusión}

Investigaciones realizadas en diversas regiones del mundo evidencian que las especies del género Aeromonas se aíslan con frecuencia en personas afectadas con procesos infecciosos intestinales y extra-intestinales ${ }^{16}$. Muchos estudios epidemiológicos han demostrado la presencia de este agente con una mayor frecuencia en heces de pacientes con trastornos gastrointestinales que en las de individuos asintomáticos pudiendo causar gastroenteritis crónica o enterocolitis autolimitada en niños y ancianos, o cursar con un cuadro clínico de fiebre, vómitos y aumento de leucocitos fecales ${ }^{6}$.

Mientras que muchos investigadores refutan el hecho de aceptar a este microorganismo como causa de enfermedad diarreica, los resultados disponibles en la literatura científica soportan la conclusión de que algunas cepas con codificación genética para determinados factores de virulencia asociados a enteropatogenicidad pueden causar gastroenteritis aguda o crónica con dosis infectantes mayores o iguales a 6-8 $\log _{10} \mathrm{ufc} / \mathrm{g}$, como lo demuestran los estudios de Moyer y Larebb 1988, quienes aislaron Aeromonas en un niño de tres meses con diarrea crónica, los de Altwegg y cols., en 1991, quienes evidenciaron este microorganismo en un adulto con gastroenteritis aguda, así como los de Moyer y cols., 2002, quienes aislaron Aeromonas en las heces de un niño de dos años con gastroenteritis aguda ${ }^{17,18}$.

Chang y cols., 2003, al realizar en Hong Kong un estudio longitudinal durante un año en pacientes con EDA que acudían a la sala de emergencia del Hospital "Príncipe de Gales" de ese país, notificaron el aislamiento de Aeromonas (9\%) como agente causal, con resultados similares a los obtenidos en esta investigación ${ }^{19}$. Yang Bai y cols., 2004, identifican Aeromonas spp en un brote de EDA ocurrido durante un ejercicio militar al sur de $\mathrm{China}^{20}$.

En la presente investigación, al establecer la asociación de Aeromonas aisladas de niños con enfermedad diarreica aguda en 17 estudios de casos y controles en Cuba, los resultados muestran que este agente bacteriano estuvo presente en una frecuencia cuatro veces mayor en niños con diarrea respecto de controles que no la tenían. Estos resultados son similares a los publicados por Albert y cols., en 1999, quienes en un estudio de casos y controles realizado en niños con EDA en Bangladesh, demostraron que esta bacteria se aisló en $74,8 \%$ de los niños sintomáticos y en $43,9 \%$ de los asintomáticos ${ }^{21}$.

De igual forma, nuestros datos concuerdan con Dallal y MoezArdalan quienes, en un estudio realizado en un hospital pediátrico de Irán, evidenciaron que los porcentajes de aislamiento de Aeromonas resultaron mayores en los niños con diarrea que en los no la presentaban, concluyendo así que algunas cepas de Aeromonas son capaces de provocar diarreas en hospederos susceptibles ${ }^{22}$.
La tipificación de las cepas a nivel de especie en este género, es de suma importancia desde el punto de vista clínico y epidemiológico, debido a la variación interespecie, en sus diferentes capacidades invasoras y enterotoxigénicas, los perfiles de susceptibilidad antimicrobiana y la distribución geográfica de cada especie ${ }^{23}$.

En 1995, Bravo y cols., publicaron un estudio sobre nuevas especies de Aeromonas aisladas en heces de niños con EDA en Cuba. Los autores demostraron la circulación de las especies $A$. caviae, A. hydrophila y $A$. veronii bv sobria y, en un menor porcentaje aislaron $A$. veronii bv veronii y $A$. schubertii ${ }^{24}$. La presente investigación corrobora la permanencia de estas especies en Cuba. Resultados similares a los obtenidos en este trabajo se describieron en Kokalta, región de la India, donde en un estudio realizado durante dos años consecutivos en niños con enfermedad diarreica aguda, se comprobó el predominio de las especies antes mencionadas ${ }^{25}$, mientras que en México, Arteaga-Garibay y cols., al estudiar cepas procedentes de pacientes con enfermedad diarreica aguda, señalaron también el predominio de $A$. caviae, $A$. hydrophila y $A$. veronii bv sobria ${ }^{26}$.

Estudios realizados por Uhnoo y por Prestel en Suecia y Austria, respectivamente, en los cuales se investigó la presencia de agentes enteropatógenos en niños con enfermedad diarreica aguda y en un grupo control, se demostró la presencia de microorganismos pertenecientes a los géneros Aeromonas, Salmonella, Shigella y E. coli en similares porcentajes ${ }^{27,28}$.

De igual forma, coincidimos con las evidencias publicadas por Soltan Dallal y cols., quienes en un estudio realizado en Teherán, India, demostraron la presencia de los mismos agentes enteropatógenos identificados en el presente estudio como principales microorganismos asociados a la $\mathrm{EDA}^{22}$.

También concordamos con los resultados publicados por Caprioli y cols., en Italia, quienes demostraron la presencia de similares agentes enteropatógenos en niños con diarrea y en el grupo control ${ }^{29}$.

Como conclusión, podemos afirmar que el presente estudio demostró que los microorganismos pertenecientes a los géneros Salmonella, Shigella, E. coli enteropatogénica y Aeromonas están asociados a la enfermedad diarreica aguda en Cuba y que este último género circula con una frecuencia significativamente mayor en niños con diarrea que en asintomáticos, siendo $A$. hydrophila, $A$. veronii bv sobria y $A$. veronii bv veronii las especies de este género más frecuentemente asociadas a esta enfermedad digestiva en Cuba.

\section{Resumen}

Los miembros del género Aeromonas son considerados patógenos importantes del tracto gastrointestinal 
en diferentes áreas geográficas. De febrero de 1985 a enero de 2005 se realizaron estudios de casos y controles en el Laboratorio Nacional de Referencia de Enfermedades Diarreicas Agudas del Instituto Pedro Kourí con el objetivo de conocer el comportamiento de los microorganismos pertenecientes al género Aeromonas en niños con diarreas en Cuba. La muestra estuvo constituida por 2.322 niños bajo 5 años de edad, ingresados por enfermedad diarreica aguda y como grupo control se estudió un total de 2.072 niños con edades y áreas geográficas similares que acudieron a los hospitales correspondientes en ese mismo período de tiempo. En el grupo de niños que presentaron diarreas, Aeromonas spp fue aislada en $166(7,15 \%)$, y en los controles fue encontrada en $35(1,76 \%)$. Al comparar la positividad para Aeromonas entre ambos grupos, la probabilidad de diagnosticar este microorganismo fue 4,28 veces mayor en los casos que en los controles (OR = 4,28; IC al 95\%: 2,96-6,20; $P<0,001)$. Las especies más frecuentemente aisladas fueron $A$. caviae, A. hydrophila, y $A$. veronii bv sobria. Otros enteropatógenos aislados en niños que presentaban diarrea fueron: Shigella spp. en 418 (18\%) $(P<0,0001$, Salmonella spp en $53(2,3 \%)(P<0,01)$,$) y$ E. coli enteropatógena en $58(2,49 \%)(P<0,05)$.

\section{Referencias}

1.- Freijoso E, Cires M, Silva L, Delgado I, Riverón R, Ramírez M. Guía para la práctica de las enfermedades diarreicas agudas. Rev Cubana Med Gen Integr 2003; 19(4): 864-5.

2.- Ortiz Bultó P L, Pérez A, Rivero A, Díaz M. Pronóstico bioclimático mensual: enero 2008. Boletín epidemiológico del IPK. 2007; 17 (52) [citado el 31/08/2008]. Disponible en: http:// www.ipk.sld.cu/bolepid3/bol52-07.htm

3.- Galindo C L, Chopra A K. Aeromonas and Plesiomonas species. En: Doyle MP, Beuch LR, editores. Food Microbiology: fundamentals and frontiers. Washington, DC: ASM Press; 2007. p. 1-56.

4.- Material safety data sheet: infectious substances. Section 1. Infectious agent. Office of Laboratory Safety, PPHB. 2001 [citado de 20 abril 2004]; Disponible en: URL: http://www.hc-sc.gc.ca/ pphb-dgspsp/msds-ftss/msds6e.html

5.- Figueras M J. Clinical relevance of Aeromonas. Rev Med Microbiol 2005; 16: 145-53.

6.- Janda J M, Abbott S L. Evolving concepts regarding the genus Aeromonas; and expanding panorama of species, disease presentations, and unanswered questions. Clin Infect Dis 1998; 27: 332-44. Review.

7.- Taneja N, Khurana S, Trhan A, Marwaha R K, Sharma M. An outbreak of hospital acquired diarrhea due to Aeromonas sobria. Indian Pediatr 2004; 41: 912-6.

8.- Nzeako B, Okafor N. Bacterial enteropathogens and factors associated with seasonal episodes of gastroenteritis in Nsukka, Nigeria. Br J Biomed Sci 2002; 59: 76-9.

9.- Vizcaya L, Carrero A, Hernández J, Nieves B, Pérez-Schael I. Origen bacteriano de la enfermedad diarreica aguda en Mérida, Venezuela. Rev Cubana Med Trop 1999; 51: 14-9.

10.- Ogunsanya P I, Rotimi V O, Adenuga A. A study of the aetiological agents of chilhood diarhoea in Lago, Nigeria. J Med Microbiol 1994; 40: 10-4.
11.- Koneman E W, Allen S D, Dowell V R, Janda W N, Sommers H M, Winn W C. Diagnóstico Microbiológico. 3ra. ed. Buenos Aires: Editorial Médica Panamericana; 1998.

12.- Martin-Carnahan A, Joseph S W. Aeromonadaceae. En: Brenner DJ, Krieg NR, Staly JT, Garrity GM, editores. The Proteobacteria, Part B, Bergey's Manual of Sistematic Bacteriology. $2^{\text {da }}$. ed. Volumen 2. New York: Springer-Verlag; 2005.p. 557-78.

13.- Furuwatari C H, Kawakami Y, Akahame T, Hidaka E, Okimura Y, Nakayama J. Proposal for an Aeroscheme (modified Aerokey II) for the identification of clinical Aeromonas species. Med Sci Res 1994; 22: 617-9.

14.- Carnahan A, Behran S, Joseph S. Aerokey II: a flexible key for identifying clinical Aeromonas species. J Clin Microbiol 1991; 29: 2843-9.

15.- Dean A G, Dean J A, Coulombier D, Brendel K A, Smith D C, Burton A H, et al. EpiInfo Version 6: a word processing, database, and statistics program for epidemiology on microcomputers. Atlanta (GA): Centers for Disease Control; 1994.

16.- Castro-Escarpulli G, Aguilera-Arreola M G, Hernández-Rodríguez C H, Arteaga-Garibay N I, Carmona-Martínez AA, Peréz-Valdespino A, et al. La identificación genética de Aeromonas una realidad y una necesidad en la microbiología diagnóstica. Bioquimia 2003; 28 (4): 11-8.

17.- Moyer NP, Larew MS. Recurrent gastroenteritis caused by Aeromonas species: a case history. J Diarr Dis Res 1988; 8: 144-5.

18.- Altwegg MG, Martinetti L, Luthy-Hottenstein J, Rohrbach M. Aeromonas associated gastroenteritis after consumption of contaminated shrimp. Eur J Clin Microbiol Infect Dis 1991; 10: 4445.

19.- Chang W S S, Ng K C, Lyon D J, Cheung W L, Rainer T H. Acute bacterial gastroenteritis: a study of adult patients with positive stool cultures treated in the emergency department in a hospital in Honk Kong. Emerg Med J 2003; 20: 335-8.

20.- Yang Bai, Ying-Chun D, Nang-Dong J L. Acute diarrhea during army field exercise in southern China. World J Gastroenterol 2004; 10 (1): 127-31.

21.- Albert M J, Faruke A S, Faruke S M, Sack R B, Mahalanabis D. Case-control study of enteropathogens associated with childhood diarrheal in Dhaka, Bangladesh. J Clin Microbiol 1999; 37: 3458-64.

22.- Soltan Dallal M M, Moezardalan K. Aeromonas spp. associated with children's diarrhoea in Tehran: a case-control study. Ann Trop Paediatr 2004; 24 (1): 45-51.

23.- Hsueh P R, Teng L J, Lee L N, Yang P C, Chen Y $\mathrm{C}, \mathrm{Ho} \mathrm{S} \mathrm{W}$, et al. Indwelling device-related and recurrent infections due to Aeromonas species. Clin Infect Dis 1998; 26:651-8.

24.- Bravo L, Monte R, Alfonso V, Cabrera N, Gómez M, Hernández R, et al. Nuevas especies de Aeromonas aisladas en Cuba. Rev Cubana Med Trop 1995; 47 (3): 215-6.

25.- Sinha S, Shimada T, Ramamurty T, Bhattacharya S, Yamasaki Y, Balakrish N G. Prevalence, serotype distribution, antibiotic susceptibility and genetic profiles of mesophilic Aeromonas species isolated from hospitalized diarrhoeal cases in Koalta India. J Med Microbiol 2004; 53: 527-34.

26.- Arteaga-Garibay R I, Aguilera M G, Navarro A, Molina J, Cravioto A, Valdespino A. El papel del flagelo lateral en la adherencia de Aeromonas spp. Difusión Internacional 2005; 30: 95

27.- Uhnoo J, Wadell G, Svensson I, OldingStenkvist E, Ekwall E, Möllby R. Aetiology and epidemiology of acute gastroenteritis in Swedish children. J Infect. 1986; 13: 73-89.

28.- Prestel E, Nadrrhal R, Wolf D, Rotter M, Hirschl A M. Enteroaggregative and enterotoxigenic Escherichia coli among isolates from patients with diarrhea in Austria. Eur J Clin Microbiol Infect Dis 1999; 18: 20912.

29.- Caprioli A, Pezzella C, Morelli R. Enteropathogens associated with childhood diarrhea in Italy. Pediatr Infect Dis J 1996; 15 : 876-83. 